\title{
Medizinstudium: Wer bleibt auf der Strecke?
}

\section{Dänische Forscher untersuchten, ob es Prädiktoren dafür gibt, welche Medizinstudenten ihr Studium nicht abschließen.}

- Um eine verlässliche Antwort zu finden, fassten sie alle zu diesem Thema publizierten Untersuchungen zusammen. Insgesamt konnten sie 13 Studien in ihre Analyse einschließen. Die Auswertung zeigte, dass Geschlecht und Alter hier keine Rolle spielen. Dagegen fanden sich Hinweise dafür, dass eine introvertierte Persönlichkeitsstruktur, schlechte Schulnoten und schwache
Leistungen zu Beginn die frühzeitige Aufgabe des Studiums wahrscheinlicher machen. Andere Faktoren schienen vor dem "Drop-out" zu bewahren: hoher sozialer Status der Eltern, Eltern die selber Ärzte sind, und problembasiertes Lernen an der Uni.

\section{Kommentar}

Dass das Medizinstudium kein Zuckerlecken ist, wissen wir alle. Hinzu kommt, dass es mit hohen Kosten verbunden ist. Könnten wir potenzielle "Drop-outs" schon vorher identifizieren, wäre es möglich, solche Personen erst gar nicht zum Studium zuzulassen.
Wirklich? Nein, natürlich wäre das eine völlig falsche Umsetzung solcher Befunde. Der richtige Weg wäre, "Risiko-Studenten" besser zu helfen, damit sie es bis zum Staatsexamen schaffen. Um dies effektiv zu bewerkstelligen, sind die Daten jedoch zu dünn und weisen zu viele Widersprüche auf. Fazit: Die Befunde sind interessant, aber praktisch kaum umsetzbar.

E. ERNST :

\section{- L. D. O'Neill, B. Wallstedt, B. Eika,}

J. Hartvigsen

Factors associated with dropout in medical education: a literature review.

Med. Educ. 2011 Mar 22. doi: 10.1111/j.13652923.2010.03898.x.

\section{Blaue Füße nach aortokoronarem Bypass}

\begin{abstract}
Fünf Wochen nach einer komplikationslos verlaufenen aortokoronaren Bypassoperation stellte sich eine 81-jährige Frau wegen einer ausgedehnten Livedo der Füße und Unterschenkel (Abbildung $A$ ) und einer Zyanose der Zehen vor (Abbildung B).
\end{abstract}

- Das Serumkreatinin war mit 5,6 mg/ dl deutlich erhöht, die geschätzte GFR betrug $46 \mathrm{ml} / \mathrm{min}$. Während des stationären Aufenthalts im Rahmen der Bypassoperation lag der Kreatininwert noch bei $1,2 \mathrm{mg} / \mathrm{dl}$ und war stabil geblieben. Differenzialblutbild, antizytoplasmatische Antikörper und Kryoglobuline waren negativ, das Komplement normal.Der Urinstatus und die Nierensonografie ergaben ebenfalls keinen pathologischen Befund.

In einer Nierenbiopsie erkannte man zahlreiche Cholesterinkristalle, die zu einer Obstruktion einer A. arcuata geführt hatten (Abb. C, Masson-Goldner Trichromfärbung). Diese Befunde sprachen für das Vorliegen von Cholesterinembolien, die zu einem Nierenversagen geführt hatten.

Man darf annehmen, dass sich aufgrund der chirurgischen Manipulation an den Koronararterien atheromatöse
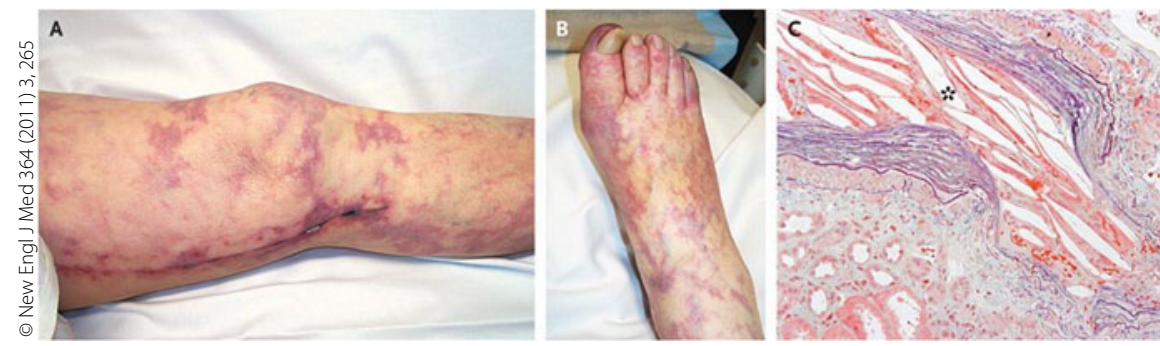

Livedo der Unterschenkel (A) und Füße (B). Cholesterinkristalle und Obstruktion einer A. arcuata im Nierenbiopsat (C).

Plaques gelöst hatten, die sich als Embolie über das arterielle Gefäßsystem verteilten und auch zu einer Obstruktion von Nierengefäßen führten. Das Auftreten der Livedo reticularis spricht auch für eine Embolie in periphere Arteriolen. Die Patientin musste im weiteren Verlauf dialysiert werden. Sie erlitt mehrere zerebrale Krampfanfälle und verstarb schließlich an einer Sepsis.

\section{Kommentar}

Das Cholesterinembolie-Syndrom wird verursacht durch den Verschluss kleiner Arterien durch Emboli von Cholesterinkristallen aus ulzerierten arteriosklerotischen Plaques. Selten erfolgt die Freisetzung der Cholesterinkristalle spontan, in den meisten Fällen gehen dem Ereignis medizinische Maßnahmen voraus wie Angiographien, Thrombolysen und gefäßchirurgische Eingriffe. Meistens ist die Bauchaorta der Ursprung der Kristalle. Die Ablagerung der Emboli erfolgt am häufigsten in den Gefäßen der Nieren, der Haut, des Magen-Darm-Trakts und der Retina. Die Trias auslösendes Ereignis, Nierenversagen und Emboliesymptome sind diagnostisch wegweisend. Im Blutbild liegt typischerweise eine Eosinophilie vor. Die Prognose der Erkrankung ist schlecht, nicht selten stellt sich eine chronische Niereninsuffizienz ein. Der Krankheitsverlauf wird durch eine Therapie mit Statinen günstig beeinflusst.

H. S. FÜESSL =

\footnotetext{
- R. D. Frank, J Velden

(Dario.Frank@sah-eschweiler.de): Cholesterol emboli after coronary bypass surgery. New Engl J Med 364 (2011) 3, 265
} 